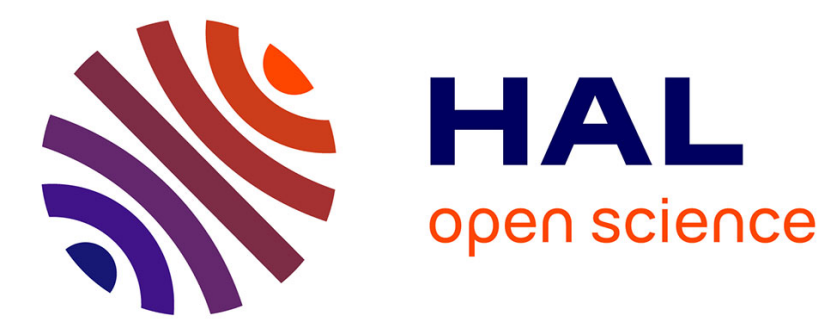

\title{
La concurrence avant la libéralisation: International Post Corporation, entreprise de transformation, 1983-1989
} Léonard Laborie

\section{To cite this version:}

Léonard Laborie. La concurrence avant la libéralisation: International Post Corporation, entreprise de transformation, 1983-1989. Entreprises et Histoire, 2021, 105. hal-03512991

\section{HAL Id: hal-03512991 \\ https://hal.science/hal-03512991}

Submitted on 5 Jan 2022

HAL is a multi-disciplinary open access archive for the deposit and dissemination of scientific research documents, whether they are published or not. The documents may come from teaching and research institutions in France or abroad, or from public or private research centers.
L'archive ouverte pluridisciplinaire HAL, est destinée au dépôt et à la diffusion de documents scientifiques de niveau recherche, publiés ou non, émanant des établissements d'enseignement et de recherche français ou étrangers, des laboratoires publics ou privés. 
Léonard Laborie, " La concurrence avant la libéralisation : International Post Corporation, entreprise de transformation, 1983-1989

Ce texte correspond à la version 1 d'un article, avant sa relecture par les coordinateurs du dossier et les éditeurs de la revue dans laquelle il a été publié : Léonard Laborie, « La concurrence avant la libéralisation : International Post Corporation, entreprise de transformation, 1983-1989 », Entreprises et histoire, n¹05, 2021, p. 52-64). L'article final fait référence. Certes moins détaillé, certaines interprétations y ont été revues après consultation de sources imprimées complémentaires et échange avec les relecteurs.

\title{
La concurrence avant la libéralisation : International Post Corporation, entreprise de transformation, 1983-1989
}

\author{
Léonard Laborie \\ CNRS, UMR Sirice
}

\section{Introduction}

«Une initiative déterminante; la plus importante que le secteur international ait connu ces 50 dernières années $»^{1}$ : c'est en ces termes que Ronald Dearing, ancien patron de Royal Mail, enjoint ses homologues de créer un nouvel organisme international, International Post Corporation, en mai 1988. L'enjeu est de donner aux postes associées « la capacité de rivaliser en créativité, en action, en possibilité de décisions, avec les entreprises privées les mieux dirigées, les plus déterminées et les mieux financées du monde des transports $»^{2}$. Dearing et le groupe d'action qu'il préside au sein de la Conférence européenne des administrations des postes et des télécommunications depuis quelques mois sont entendus. International Post Corporation a bien vu le jour, en janvier 1989. Mais qui, aujourd'hui, s'en souvient?

En revenant sur les origines d'International Post Corporation, cet article éclaire les motivations, la forme et la déception d'une mobilisation postale collective, inédite et largement oubliée ${ }^{3}$. Grâce aux archives de l'administration française, particulièrement active sur ce projet, il présente de l'intérieur les réflexions et négociations qui ont conduit une quinzaine de postes européennes rejointes par celles du Canada, des Etats-Unis, de l'Australie et du Japon à s'allier pour fonder une entreprise commune. Fruit et, espérait-on, véhicule d'une entreprise coopérative de transformation de ses membres, International Post Corporation devait préserver un ordre postal séculaire en vertu duquel chaque poste était maîtresse chez elle ${ }^{4}$, en les rendant collectivement compétitives face à la nouvelle concurrence sur le créneau des relations internationales de ceux que l'on commence à l'époque à dénommer les « intégrateurs », comme DHL, Federal Express ou UPS.

\footnotetext{
1 «Résumé du rapport final du groupe d'action CEPT », mai 1988. AN 19990382/14.

2 Idem.

${ }^{3}$ En témoigne la très mince littérature existante à son sujet. Deux ouvrages l'abordent, à la marge : Camille Allaz, Une histoire de la Poste dans le monde, Paris, Pygmalion, 2013 et James I. Campbell, The Rise of Global Delivery Services. A Case Study in International Regulatory Reform, Washington, JCampbell Press, 2001.

${ }^{4}$ Léonard Laborie, L'Europe mise en réseaux. La France et la coopération internationale dans les postes et les télécommunications (années 1850-années 1950), Bruxelles, Peter Lang, coll. Enjeux internationaux, 2010.
} 
Léonard Laborie, « La concurrence avant la libéralisation : International Post Corporation, entreprise de transformation, 1983-1989

L'exploration transnationale et cognitive -attentive à l'élaboration de représentations du monde partagées-, des origines de l'entreprise International Post Corporation enrichit notre connaissance de l'histoire de l'Europe des communications postales, qui reste moins connue que l'Europe des communications électroniques ${ }^{5}$. Elle documente la double transition de ce secteur, passé des années 1970 aux années 1990 d'un cadre réglementaire monopolistique à un cadre concurrentiel, avec des administrations qui sont devenues des entreprises, selon des chronologies, des articulations et des modalités qui, malgré des tendances communes, varient d'un pays à l'autre. Essentiellement décrite du point de vue des sciences politiques, économiques et juridiques d'une part, et sous l'angle des trajectoires nationales d'autre part ${ }^{6}$, la libéralisation postale peu spectaculaire car progressive et finalement moins perceptible par les citoyens au quotidien que dans le secteur des télécommunications, a moins attiré l'attention des chercheurs. Mark Thatcher a toutefois montré que les puissants facteurs d'ouverture des marchés des télécommunications qu'étaient l'évolution technique et la déréglementation sur les marchés américain et britannique n'ont pas été à l'œuvre dans le domaine postal ${ }^{7}$. On pourrait nuancer le premier point en rappelant notamment que la combinaison d'un transport aérien moins cher et plus rapide (grâce au moteur à réaction et à des appareils comme le Boing 747, datant de 1969), et de réseaux de télécommunication numérisés permettant le suivi des objets, a considérablement fait évoluer la donne dans la circulation internationale des lettres et autres marchandises légères. Pour Thatcher, le principal facteur d'ouverture des marchés postaux nationaux à la concurrence a été, en quelque sorte, idéologique, à travers la déréglementation communautaire mise en œuvre à partir de la seconde moitié des années 1990. Sans nier l'importance du livre vert sur le développement du marché unique des services postaux de 1992 et les directives qui ont suivi à partir de 1997, le présent article propose de décaler la focale chronologique et interprétative, en se déplaçant dans les années 1980, du côté des responsables des services postaux concernés. Il ne dit pas que les responsables des postes ont voulu libéraliser, au contraire, mais il démontre qu'ils se sont convaincus ensemble, dès cette époque, qu'ils étaient des acteurs opérant sur un marché concurrentiel ou appelé à le devenir, et que, ce faisant, ils se sont engagés d'eux-mêmes sur une voie de transformation susceptible de remettre en cause l'ordre établi. L'article rejoint ainsi les études récentes qui tendent à réviser la chronologie de la libéralisation sectorielle amorcée en Europe, en montrant que la concurrence est présente dès avant qu'elle ne soit légalement instituée par la voie de directives ${ }^{8}$.

La première partie de l'article examine le développement d'opérateurs privés de transport accéléré international de courrier et de marchandises en Europe à partir de la seconde moitié des années 1970 et le tournant que représentent les années 1983-1984 dans la confrontation des postes européennes à cette concurrence. La deuxième partie décrit la riposte qui se met en

\footnotetext{
${ }^{5}$ Pascal Griset, Andreas Fickers, Communicating Europe. Technologies, Information, Events, London, Palgrave Macmillan, 2020.

${ }^{6}$ Voir les travaux de Michael A. Crew et Paul R. Kleindorfer, précisément initiés dans le contexte décrit dans cet article, depuis le volume collectif Competition and innovation in postal services, Boston, Kluwer, 1991, ainsi que les publications de James Campbell, déjà cité. Ce dernier, juriste américain, fut secrétaire général de l'International Courier Conference puis de l'International Express Carriers Conference (1985-1989), principales associations des concurrents des postes.

${ }^{7}$ Mark Thatcher, Internationalisation and Economic Institutions: Comparing the European Experience, Oxford, Oxford University Press, 2007 (chapitre "Liberalising the Letter: The Reform of Postal Services").

${ }^{8}$ Christian Henrich-Franke, "EC competition law and the idea of "open networks" (1950s1980s)", Internet Histories, vol. 4, n², 2020, p. 125-141.
} 
Léonard Laborie, "La concurrence avant la libéralisation: International Post Corporation, entreprise de transformation, 1983-1989

place entre 1984 et 1987, à la fois commerciale, logistique et organisationnelle. La troisième partie explique comment, sur ces bases, l'idée d'un nécessaire approfondissement de la coopération a conduit les postes partenaires à fonder International Post Corporation en 1989.

\section{La morsure de la concurrence}

Plusieurs événements s'enchainent dans la seconde moitié des années 1970, qui inaugurent une confrontation à la fois commerciale et juridique entre postes européennes et opérateurs privés, originaires principalement des Etats-Unis, d'Asie et d'Australie. Parmi la multitude de compagnies qui se créent depuis la fin des années 1960 pour exploiter les nouvelles possibilités ouvertes par le transport aérien de masse et pour répondre aux besoins, frustrés ou ignorés, des entreprises en matière d'acheminement très rapide, par voie aérienne, de courriers -on parle alors de coursiers- ou de colis -on parle alors d'expressistes-, ce sont DHL et Federal Express (Fedex) qui connaîtront le plus bel avenir'. En s'installant sur le sol européen dès 1974, la première précède de dix ans la seconde. L'entrée en scène de la Commission européenne en 1983 suivi de l'arrivée de Federal Express en 1984 marquent un tournant.

\section{DHL \& Co}

DHL, fondée en 1969 par trois étudiants sur la côte Ouest, se spécialise sur le transport de documents par-dessus l'océan Pacifique -d'abord vers les îles de Hawaï et de Guam. Au départ, les documents sont chargés à bord des appareils en tant que bagages. À Hong Kong, colonie britannique en pleine expansion économique au large de la Chine continentale, les autorités réagissent vertement à l'installation du coursier en $1972^{10}$. Oscillant entre saisies, menaces et tolérance, elles abandonnent finalement les poursuites pour enfreinte au monopole postal en 1975, voyant l'intérêt pour le territoire d'être doté d'un opérateur qui le connecte au reste du monde pour l'acheminement physique rapide de l'information ${ }^{11}$.

Car entretemps, la petite entreprise s'est développée à grande vitesse postant ses « cowboys $»^{12}$ des airs autour du Pacifique, et jusqu'en Europe et en Afrique, pour offrir un service global. En Europe, où il existe des coursiers locaux (Airport Couriers, City Courier, IML, etc. $)^{13}$, elle s'installe d'abord à Londres en 1974, puis en Ecosse et sur le continent, avec l'ouverture de bureaux à Amsterdam et Paris en 1975. L'année suivante, c'est un vrai poids lourd qui débarque en République fédérale d'Allemagne, United Parcel Service (UPS) ${ }^{14}$. Messager terrestre américain, «Big Brown » entend diversifier son activité en développant le transport express de colis internationaux. Elle s'installe à Cologne, en Rhénanie, en plein cœur économique de l'Europe de l'ouest.

Face à cette conjonction de facteurs, les postes décident dans le courant des années 1970 de mettre en place un nouveau service international, l'Expedited (ou Express) mail service (EMS), courrier accéléré international en français ${ }^{15}$. Inspiré jusque dans son acronyme du

\footnotetext{
${ }^{9}$ Allaz, Histoire de la Poste dans le monde, p. 616-620.

10 Témoignage de Po Chung co-fondateur de DHL International, dans Po Chung, Roger Bowie, DHL. From Startup du Global Upstart, Berlin, De Gruyter, 2018, p. xv-xvii.

${ }^{11}$ Idem, «DHL chronology » (hors texte) et p. 39-40. Camille Allaz date l'octroi de licences aux coursiers internationaux à Hong Kong de 1976. Allaz, Histoire de la Poste dans le monde, p. 621.

12 Idem, p. 39.

${ }^{13}$ Campbell, The Rise of Global Delivery Services, p. 3.

${ }^{14}$ Niemann Greg, Big Brown : the Untold Story of UPS, San Francisco, John Wiley \& Sons, 2010, p. 161-179.

${ }^{15}$ EMS Actualités, $\mathrm{n}^{\circ}$ spécial sur le colloque EMS 1986, janvier 1987.
} 
Léonard Laborie, « La concurrence avant la libéralisation : International Post Corporation, entreprise de transformation, 1983-1989

service de la poste américaine créé dans la foulée de son changement de statut en 1970 -USPS est un service du gouvernement, indépendant, et non plus un département du cabinet-, l'EMS est une réponse coopérative à l'essor des coursiers. Si ses caractéristiques générales sont fixées au sein de l'Union Postale Universelle (UPU) à travers des discussions multilatérales, l'exécution opérationnelle fait l'objet d'accords bilatéraux. Objectif : raccourcir au maximum les délais d'acheminement et de distribution.

Cette réaction témoigne d'une volonté d'adaptation de la part des postes, qui ont engagé depuis la fin des années 1960 un premier virage commercial, à la demande de leurs tutelles politiques -souvent elles-mêmes interpelées en Europe par la branche télécommunications, lassée d'avoir à combler les déficits postaux. Les services domestiques EMS aux Etats-Unis, Datapost au Royaume-Uni, Postadex en France ont commencé à répondre aux besoins exprimés par les utilisateurs ${ }^{16}$. Mais ce virage, qui s'accompagne d'un côté d'une quête de productivité, avec notamment un investissement dans des machines automatiques de tri pour faire face à la hausse du trafic, et de l'autre d'une réflexion sur l'avenir du lien avec les services de télécommunications, provoque des inquiétudes chez les agents. Des grèves de grande ampleur perturbent les services sur la période ${ }^{17}$. C'est aussi dans ce contexte que se développent les alternatives privées ${ }^{18}$.

\section{La décision de la Commission des Communautés européennes}

Si les postes réagissent déjà sur le plan commercial, leur principale action est d'ordre juridique $^{19}$. En France, l'administration impose en 1980 à DHL de lui verser une compensation pour chaque pli pris en charge sur le territoire national. Après l'élection de François Mitterrand à la présidence de la République en mai 1981 et l'installation d'un gouvernement socialiste, la concurrence faite au service public est combattue plus frontalement. L'administration postale exige désormais une limitation drastique de l'activité de DHL, qui ne pourra se faire qu'au départ ou à destination de Paris. Face au refus de l'entreprise américaine, le ton monte. Un peu comme à Hong Kong dans la première moitié des années 1970, des contrôles et des saisies sont effectuées dans ses bureaux et dans les aéroports par les douanes. Ce bras de fer se joue au même moment à travers toute la Communauté européenne, sauf en Grande-Bretagne où Margaret Thatcher a décidé dès 1981 d'ouvrir le marché postal à la concurrence pour le courrier express facturé plus d'une livre à l'unité. Au niveau de l'UPU, les postes lancent une étude commune sur les moyens juridiques à leur disposition pour faire respecter leur monopole, qui, selon des configurations locales différentes, est partout sous pression ${ }^{20}$.

\footnotetext{
${ }^{16}$ Allaz, Histoire de la Poste dans le monde, p. 622. Sur Postadex : Léonard Laborie, Sébastien Richez, Attention fragile! Economie et politique de la messagerie postale en France, XIXe-XXe s., Bruxelles, Peter Lang, 2020, p. 284.

${ }^{17}$ Vincent Bouget, «La grève des PTT de l'automne 1974 et les médias », Les Cahiers pour l'histoire de La Poste, $\mathrm{n}^{\circ}$ 1, Paris, 2003.

${ }^{18}$ Le rapprochement est fait, au moins rétrospectivement, par les responsables des postes euxmêmes. Voir notamment : Gerard Harvey, «Unipost. A Postal Strategy in a Global Market”, in Walpurga Speckbacher (éd.), Die Zukunft der Postdienste in Europa: Proceedings der internationalen Konferenz „Die Zukunft der Postdienste in Europa“ Bonn, 25.-26.10.1990, Springer, 2013, p. 179-185, p. 181.

${ }^{19}$ Sur ce qui suit: Campbell, The Rise of Global Delivery Services, p. 146 et suivantes.

${ }^{20}$ Etude 522 du Conseil consultatif des études postales de l'UPU, menée sur la période 19791984.
} 
Léonard Laborie, « La concurrence avant la libéralisation : International Post Corporation, entreprise de transformation, 1983-1989

Alertée, la Commission des communautés européennes ouvre en juin 1983 une enquête sur le traitement des coursiers internationaux par les monopoles postaux ${ }^{21}$. DHL, marqué par les effets négatifs de la désunion dans les négociations sur le monopole postal en Argentine et au Soudan, initie la création d'un organisme représentatif de toute l'industrie, l'International Courier Conference (ICC) ${ }^{22}$, pour faire front commun et alimenter la Commission en études prouvant l'importance du service fourni aux entreprises, non satisfaites par l'offre postale dans les relations transfrontalières. La peur commence à changer de camp. Ce sont désormais aux postes de faire la preuve qu'elles ne contreviennent pas aux règles du Traité de Rome en contraignant l'activité internationale des coursiers. Une affaire assez similaire est alors connue de tous, qui a vu quelques mois plus tôt la Commission imposer le droit de la concurrence européen à l'opérateur britannique de télécommunications ${ }^{23}$. Travaillées par l'ICC, les autorités allemandes reconnaissent courant 1984 la légalité des services internationaux offerts par les opérateurs privés à la lumière de la jurisprudence européenne ${ }^{24}$. La Bundespost doit cesser ses poursuites.

\section{Prise de conscience}

Il devient clair pour les autres administrations postales européennes que le monopole de droit dont elles jouissent sur le plan national ne les immunisera pas forcément de la concurrence sur le plan international. Or il est un service sur lequel elles opèrent depuis longtemps en situation concurrentielle, le colis postal international, dont la « désaffection ${ }^{25}$ depuis le milieu des années 1970 augure du pire.

Le service national des colis postaux n'est que rarement partie intégrante du monopole, et sa déclinaison à l'échelle internationale proposée depuis les années $1880^{26} \mathrm{n}$ 'est qu'une solution parmi d'autres à la disposition des expéditeurs et destinataires de marchandises légères. Les expressistes ont donc sur ce créneau toute latitude pour agir, pour autant qu'ils ne prennent pas en charge de correspondance. DHL intègre cette activité et complète ainsi ses chargements au tournant des années 1980, alors qu'elle l'avait laissé de côté jusqu'alors pour ne pas avoir à s'embarrasser de formalités douanières.

Une première enquête réalisée par l'administration postale française montre que, par rapport à la concurrence, ses clients se plaignent de délais d'acheminement trop longs, de formalités de dépôt trop complexes pour un coût relativement élevé27. Plus tard, d'autres enquêtes révèleront que le prix est bien moins déterminant que la garantie des délais, et la fiabilité. Le problème pour la poste française comme pour ses homologues est qu'en grossissant sur ce marché déjà ouvert de la messagerie internationale, les nouveaux entrants s'étoffent et deviennent plus menaçants sur celui des courriers urgents.

Lorsque la Commission ouvre son enquête, le rapport de force paraît certes encore très déséquilibré. En volume, les coursiers sont minuscules avec leurs 4 millions d'articles transportés en Europe, à côté des géants postaux qui s'en échangent plus de 2 milliards à

\footnotetext{
${ }^{21}$ Campbell, The Rise of Global Delivery Services, p. 148-152.

${ }^{22}$ Idem, p. 9.

${ }^{23}$ Idem, p. 151.

${ }^{24}$ Idem, p. 154-155.

${ }^{25}$ Mouzin, service des Affaires internationales, direction générale des Postes, « Recensement des comptes-clients colis postaux », 27/09/1982. Archives nationales (Pierrefitte-sur-Seine), 20020509/11.

${ }^{26}$ Léonard Laborie, « Global commerce in small boxes: parcel post, 1878-1913 », Journal of Global History, 10/2, 2015, p. 235-258.

${ }^{27}$ « Recensement des comptes-clients des colis postaux et utilisation de ses résultats pour l'exploitation commerciale », sd. AN 20020509/11.
} 
Léonard Laborie, « La concurrence avant la libéralisation : International Post Corporation, entreprise de transformation, 1983-1989

travers les frontières -et encore n'est-ce là qu'une part infime de leur trafic total ${ }^{28}$. Mais on prend conscience que la dynamique est du côté d'entreprises performantes, pour certaines aux moyens financiers importants, qui commencent à bénéficier de soutiens politiques et à se faire connaître de l'opinion.

De l'autre côté de l'Atlantique, Federal Express connaît ainsi une croissance phénoménale sur le marché des petits colis urgents ${ }^{29}$. Son modèle révolutionnaire de réseau de transport aérien de nuit se révèle très performant depuis son lancement en 1973. Les colis sont collectés chez les expéditeurs et chargés en fin de journée sur des avions qui convergent vers une plateforme centrale $(h u b)$, à Memphis, pour y échanger nuitamment leur cargaison, triée et répartie sur place. Revenus au point de départ au petit matin, les avions sont déchargés et les marchandises acheminées par la route jusqu'à leur destinataire. Simple et efficace. Federal Express ouvre une liaison transatlantique en 1984, et prend consécutivement pied en Europe, en RFA d'abord. Là, rien ne l'empêche de s'occuper aussi des documents.

\section{Une riposte commune}

Les postes des pays industrialisés, en Amérique du Nord, en Europe de l'ouest, au Japon et en Australie, concentrent l'essentiel du trafic international de lettres et de marchandises. On estime à l'époque que 7 colis postaux internationaux sur 10 sont échangés entre pays membres de la Conférence européenne des administrations des postes et des télécommunications (CEPT) $)^{30}$. Aussi est-ce dans cette instance de coordination administrative légère, dotée d'un simple secrétariat tournant, élargie pour l'occasion aux partenaires des autres continents, que s'organise un début de riposte. Trois chantiers sont ouverts.

\section{Connaître le marché}

Le premier chantier relève de l'étude de marché. Si chaque poste a bien depuis chez elle une vision du problème et des opportunités, manque une vue d'ensemble, commune, du paysage concurrentiel, jusqu'alors impensé.

La CEPT, qui fait ce constat début 1984 et estime ne pas avoir les ressources internes, décide à l'automne de passer commande à un cabinet externe ${ }^{31}$. Fin 1986, l'étude n'est toujours pas lancée... Discutées de loin en loin, les spécifications ont dû être plusieurs fois modifiées. Beaucoup trop coûteuse pour les moyens qu'étaient prêts à mettre en commun les postes, l'analyse quantitative un temps envisagée est finalement remplacée en 1987 par une approche plus qualitative, centrée sur 150 à 200 grandes entreprises européennes de 19 pays consommatrices de services postaux internationaux. Les postes font ainsi l'expérience des limites de leur capacité de réaction collective. L'épisode ne sera pas sans conséquence, au moment de réagir face aux résultats calamiteux de l'étude. Mais en attendant, deux autres chantiers, plus opérationnels, sont lancés.

\section{Eurocolis, un colis postal « à qualité de service améliorée »}

\footnotetext{
${ }^{28}$ Campbell, The Rise of Global Delivery Services, p. 147.

${ }^{29}$ Allaz, Histoire de la Poste dans le monde, p. 618-620.

${ }^{30}$ Mouzin, Service des affaires internationales, Direction Générale des Postes, 19/10/1984.

AN 19960443/2.

${ }^{31}$ D. G. Foot, Royal Mail Letters, 01.09.1987. 19990382/14.
} 
Léonard Laborie, " La concurrence avant la libéralisation : International Post Corporation, entreprise de transformation, 1983-1989

Le deuxième chantier est ouvert par la France et par la Suisse ${ }^{32}$. L'idée est d'occuper un « créneau ${ }^{33}$ laissé libre par les opérateurs privés en créant un service de messagerie rapide, intermédiaire entre le non-urgent (colis postal standard) et l'express. Le projet de « colis postal européen » est validé en mai 1984 lors d'une réunion extraordinaire des directeurs généraux des Postes de la CEPT à Bordeaux ${ }^{34}$. Concrètement, on fait avec les moyens du bord, en créant une nouvelle marque pour redorer un service existant, le colis postal avion jusqu'à $20 \mathrm{~kg}$, amélioré à la marge -promesse de meilleurs délais d'acheminement par le recours au moyen de transport le plus rapide et l'établissement de positions de travail dédiés dans les centres de traitement ; priorité de dédouanement ; indemnité plus importante en cas de perte ou spoliation. Ni l'enlèvement à domicile, jugé trop difficile à mettre en œuvre, ni la garantie de délais, qui ouvriraient à des droits d'indemnisation en cas de retard, ne sont encore à l'ordre du jour. La preuve de remise est envisagée moyennant surtaxe. Il est aussi prévu de faire une fleur aux clients, en les autorisant à joindre à l'envoi des plis et documents de correspondance, jusqu'alors bannis pour protéger le trafic des lettres internationales, beaucoup plus rémunérateur rapporté à leur poids. Le tout sans augmentation de tarif. Les tests démarrent lentement en mars 1985, entre la France et la Suisse. Les résultats étant satisfaisants, le service peut ouvrir officiellement le $1^{\text {er }}$ octobre entre la France, la Suisse, les Pays-Bas, la Suède, le Royaume Uni et le Luxembourg ${ }^{35}$. Beaucoup restent hésitants : les Danois, les Belges, les Italiens, et plus grave encore les Allemands manquent à l'appel, ces derniers estiment les Français, « pour des raisons juridiques et de personnes ${ }^{36}$. Or il est évident qu' «il n'est pas possible de mettre en place un produit postal à vocation européenne sans la participation de la RFA $»^{37}$, plaque tournante concentrant plus de $20 \%$ du trafic intraeuropéen, et $20 \%$ du courrier transatlantique. Un travail de conviction a lieu au sein de la CEPT, qui prend du temps et de l'énergie. Au fil des mois, l'offre de destination s'enrichit. La RFA finit par participer début 1988. Mais on reste loin de l'effet d'affichage escompté : derrière la marque, le réseau présente pendant longtemps des trous qui en font une solution peu convaincante pour les gros expéditeurs, qui ont besoin d'un partenaire capable de leur ouvrir toutes les destinations. Les délais de livraison, variable clé, restent relativement flous. Les postes n'arrivent pas à s'engager sur mieux qu'une fourchette de remise trois à cinq jours après le dépôt. Pendant ce temps, une nouvelle concurrence émerge, qui sans se placer sur le créneau de l'express, travaille les colis groupés avec des délais garantis. Nombre de transporteurs confrontés à une conjoncture peu favorable se diversifie sur le marché du petit colis $^{38}$.

Carte réponse promotionnelle pour l'Eurocolis (1988).

\footnotetext{
32 «La France est à l'origine de cette idée ». Mouzin, Service des Affaires internationales, DGP, note manuscrite pour M. Limat, 23.12.86. AN 19990382/14.

33 «Cahiers des charges. Campagne Courrier. Communication spécifique à l'Eurocolis », 06/07/1988. AN 20020509/21.

${ }^{34} \mathrm{R}$. Montandon, « Compte rendu de la réunion restreinte tenue à Bâle le 5 octobre 1984 », Berne, 12/10/1984. AN 19960443/2.

35 «L'Eurocolis - Stratégie d'ensemble pour le transport postal de marchandises - Point 5 de l'ordre du jour », Direction des services courrier, 02/09/1987. AN 19990382/14.

${ }^{36}$ Mouzin, Service des Affaires internationales, DGP, note manuscrite pour M. Limat, 23/12/86. AN 19990382/14.

37 Idem.

38 « Cahiers des charges. Campagne Courrier. Communication spécifique à l'Eurocolis », 06/07/1988. AN 20020509/21.
} 
Léonard Laborie, « La concurrence avant la libéralisation : International Post Corporation, entreprise de transformation, 1983-1989

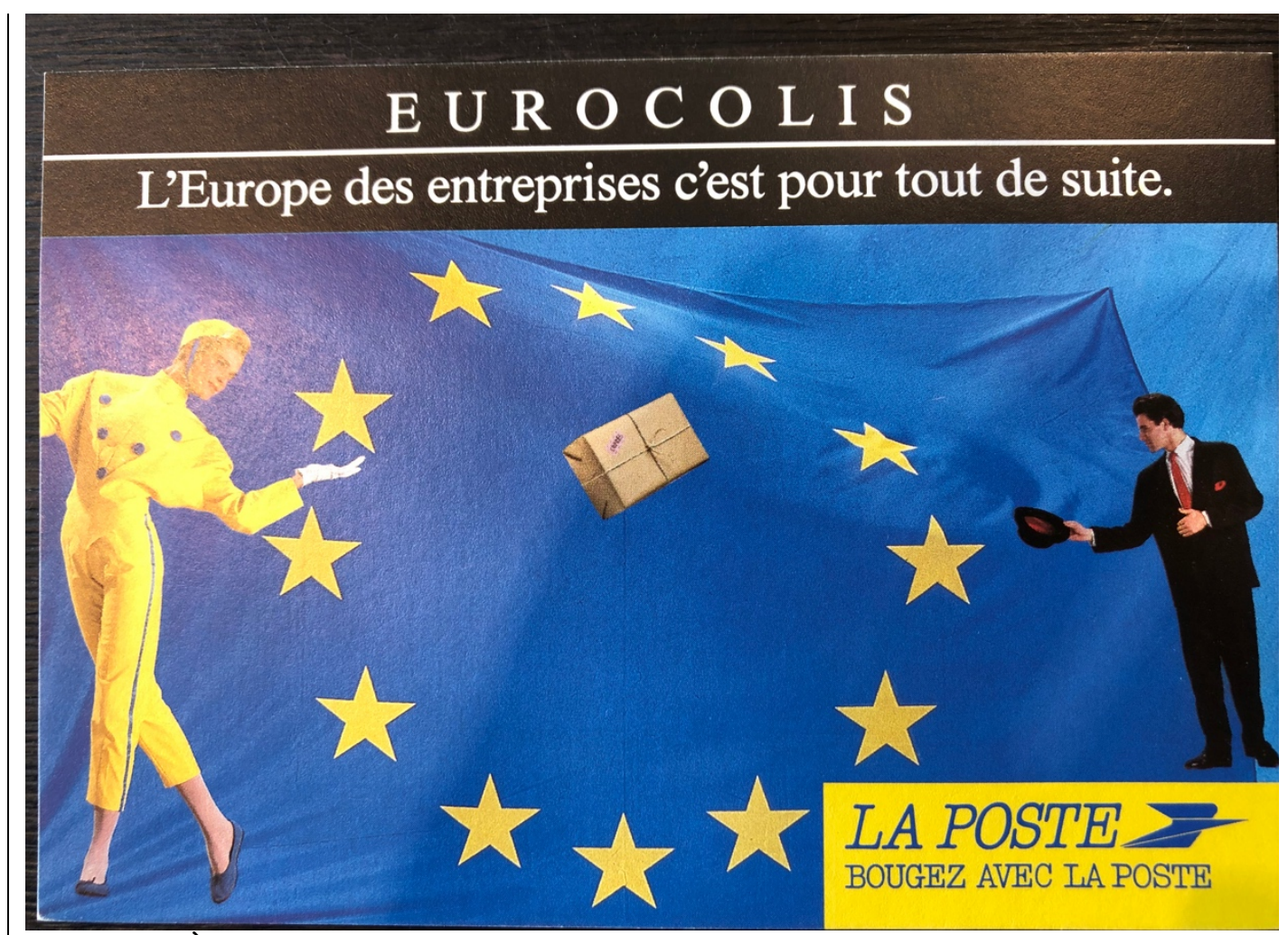

Légende : Á l'automne 1988, la Poste lance une campagne de promotion pour l'Eurocolis, constatant qu'il reste méconnu du public et mal « vendu » au guichet. Trois raisons à cela : il est long à traiter (10 minutes en moyenne), moins valorisant que Chronopost et ne donne lieu à aucune prime d'intéressement ${ }^{39}$.

Source : AN 20020509/21.

\section{EMS-International Post Corporation : une société coopérative pour gérer le hub de Bruxelles}

Le troisième chantier est plus ambitieux. Il s'agit pour les postes de se mettre en capacité de répondre frontalement à la concurrence des coursiers et expressistes, en proposant un service accéléré qui tienne ses promesses. Faute d'un plan d'ensemble, c'est au terme d'une série de décisions, prises comme par effet d'engrenage, que les partenaires se rapprochent au sein d'une nouvelle structure, EMS - International Post Corporation, en 1987.

Alors que Federal Express ouvre en 1985 de nouveaux bureaux à Paris, en Suisse et inaugure son siège européen à Bruxelles, et que DHL crée un tout nouveau hub dans cette même ville (Zaventem $)^{40}$, les postes réunies au sein de la CEPT commencent par réorganiser le réseau aérien de nuit qui relit les grandes métropoles européennes. La plateforme centrale est déplacée de Rotterdam à Bruxelles. Un barreau desservant la côte Est des Etats-Unis et du Canada est mis en place pour capter ce flux transatlantique stratégique sur le plan commercial.

Comme tout cela ne suffit pas, on décide bientôt de revoir la gouvernance même du réseau. Un comité directeur EMS réunissant les postes associées est chargé à partir de juin 1986 de superviser le réseau et, point qui s'avère capital pour convaincre les clients, de le doubler

39 « Cahiers des charges. Campagne Courrier. Communication spécifique à l'Eurocolis », 06/07/1988. 20020509/21.

40 «DHL restera-t-elle à Zaventem ? », Le Soir, 30/12/2003. Chronologie de l'implantation de Fedex en Europe: https://www.fedex.com/fr-fr/about/company-info/history.html (consulté le 18 mai 2021). 
Léonard Laborie, « La concurrence avant la libéralisation : International Post Corporation, entreprise de transformation, 1983-1989

d'une infrastructure informatisée de suivi (tracing) et de pistage (tracking) ${ }^{41}$. Mais faute de règles de vote claires, le comité se montre incapable de prendre des décisions, ne serait-ce que pour répartir les charges entre associés. John Payne, qui dirige Datapost, propose de donner plus d'autonomie au comité par rapport aux directeurs généraux, auxquels il doit toujours rapporter ${ }^{42}$. La poste française tire aussi la sonnette d'alarme. En octobre 1986, le directeur général adjoint Guy Meynié livre à ses partenaires un diagnostic lucide : le service EMS n'est pas au niveau des concurrents, qu'il s'agisse de rapidité, de fiabilité ou d'universalité. Il faut, selon lui, que « les services postaux agissent plus vite, agissent mieux, agissent ensemble, et agissent autrement pour distinguer l'EMS des autres produits postaux $\gg^{43}$. Sans le dire aussi clairement, il propose de suivre la voie française, qui a vu l'administration créer un an tout juste auparavant une filiale de droit privé, la Société française de messagerie internationale (SFMI), en s'associant avec un transporteur, TAT Express. Cette filiale permet de traiter l'EMS à part, en s'appuyant sur la maison mère mais en développant autant que nécessaire et possible ses propres capacités de traitement, de transport et de distribution -qui la placent, expliquera plus tard son fondateur, Guy Meynié, à l'abri, des perturbations générées par les mouvements sociaux ${ }^{44}$. Sous la marque EMS Chronopost, le service rencontre un vrai succès dès sa première année d'exercice sur le marché des liaisons intérieures. Il reste en revanche à la peine sur les liaisons internationales. La faute selon Meynié tient moins au réseau aérien de nuit qu'à la prise en charge qui suit, à la réception, par les postes destinatrices. Il se plaint ainsi sans détour auprès de son homologue britannique, John Payne : un envoi accéléré sur dix vers le Royaume Uni donne lieu à réclamation, et quand la SFMI envoie un télex pour savoir ce qu'il en est, un message sur sept seulement reçoit une réponse de Datapost ${ }^{45}$. « The situation seems worrying", conclut-i $1^{46}$.

Décision est prise d'organiser à Paris en mars 1987 une réunion à haut niveau entre postes les plus concernées. Gérard Delage, directeur général français, invite ses homologues d'Allemagne, des Etats-Unis, de Belgique, du Canada, de Grande-Bretagne, d'Irlande, du Japon, des Pays-Bas, de Suède et de Suisse. Des émissaires sont envoyés chez certains partenaires clés pour préparer la réunion : Belgique, Grande-Bretagne et Suède reçoivent la visite de MM. Lloret, Migone et Limat pour les convaincre de renforcer la coopération dans le domaine du courrier accéléré international. Il faut, s'agissant du hub, les convaincre de la " nécessité d'une structure permanente s'occupant des problèmes d'exploitation et de suivi $»^{47}$. Au-delà : Il faut affirmer à vos interlocuteurs que nous piétinons et qu'il faut que chacun s'engage plus ferme sur la qualité des services rendus (...) sur la qualité des informations, des réponses aux réclamations, des litiges. Là seul un tracing permettant une proof delivery peut nous sortir d'un mauvais pas ; actuellement malgré le REN [réseau européen de nuit] on n'est pas assez performants ${ }^{48}$.

La réunion de Paris porte ses fruits. Les directeurs s'engagent à créer une structure permanente, qui aura en charge de superviser la plateforme de Bruxelles, et de faire avancer

\footnotetext{
${ }^{41}$ John Payne, à Monsieur Gillet, inspecteur général des Postes belges, « Comité directeur du REN », 18.12.1986. 19990382/14.

42 Idem.

${ }^{43}$ EMS Actualités, $\mathrm{n}^{\circ}$ spécial sur le colloque EMS 1986, janvier 1987.

${ }^{44}$ Guy Meynié, «Réunion des directeurs généraux à Copenhague les 9, 10 et 11 septembre $1987 \gg .19990382 / 14$

${ }^{45}$ Guy Meynié, SFMI Chronopost, à J. Payne, general manager Datapost, 13/02/1987. AN $19990382 / 14$.

46 Idem.

47 « Note pour MM. R. Limat, F. Migone, G. Lloret », sans date ni auteur. AN 19990382/14.

${ }^{48}$ Idem.
} 
Léonard Laborie, « La concurrence avant la libéralisation : International Post Corporation, entreprise de transformation, 1983-1989

tous les dossiers liés ${ }^{49}$. L'administration française, qui a initié le mouvement, se voit confier en la personne de Francis Migone, chef du service des Affaires internationales, la présidence du groupe de travail chargé de faire aboutir le projet.

Dès lors, les réunions du groupe de travail, d'une task force dédiée et des directeurs généraux se succèdent avec empressement. À Londres en mai 1987, les directeurs généraux fixent le statut de la structure ${ }^{50}$. La création d'une filiale de droit privée est écartée, incompatible avec les marges de manœuvre légales, et sans doute surtout politiques, des différents partenaires. L'association internationale est jugée à l'inverse trop lâche. On se tourne alors vers une société coopérative de droit belge qui pourra « en Belgique comme à l'étranger accomplir tous actes et opérations nécessaires ou utiles à la réalisation de son objet $»^{51}$. À Copenhague six mois plus tard, les directeurs se retrouvent pour déterminer le nom de la société, les actionnaires et la gouvernance. Inspiré de celui qu'a pris la poste canadienne, qui a changé de statut en 1981 -le Post Office Department est devenu Canada Post Corporation, société publique autonome-, le nom sera : EMS - International Post Corporation (IPC).

« International », et pas « European » car les Européens n'y sont pas seuls. L'enjeu est bien de pouvoir proposer un service global, conforme aux attentes des clients. Neuf postes (Belgique, France, Grande-Bretagne, Irlande, Finlande, Suède, Norvège, Etats-Unis et du Canada) s'engagent à prendre des parts de la société, qui doit être présentée à la presse le 12 novembre 1987. D'autres promettent de s'adjoindre au groupe, mais plus tard (Danemark, Pays-Bas, Portugal, RFA). Tandis que la Suisse reste ostensiblement en retrait, le Luxembourg, la Turquie, la Grèce et l'Espagne se disent intéressés. La direction d'EMS - IPC est confiée au Suédois Bengt Holst.

Au moment même où les partenaires prennent ces décisions, ils reçoivent l'étude attendue depuis 1984 et voient l'Acte unique européen entrer en vigueur, qui promet un marché européen sans frontière pour 1992. Ils en concluent que l'EMS - IPC n'est qu'une étape.

\section{International Post Corporation, redux}

Toutes les réunions qui se tiennent au sein de la CEPT à partir de l'été 1987 transpirent l'idée que la survie des monopoles postaux est engagée. Le diagnostic fait l'unanimité. Quant au remède, c'est moins simple.

\section{Un péril mortel}

Le rapport remis par le cabinet britannique SRU confirme la baisse décennale du trafic postal international -moins $10 \%$ pour les lettres et moins $1 \%$ pour les colis, entre 1976 et $1985^{52}$. Du fait de la mauvaise qualité du service postal, et de son incapacité à évoluer rapidement ${ }^{53}$, les clients ont remplacé les lettres par le téléphone, en attendant d'être saisis comme aux EtatsUnis par la fièvre du fax produit à bas prix au Japon ${ }^{54}$, et ils ont confié leurs colis aux transporteurs privés. Sur ce marché tiré par l'internationalisation des chaines de production et le « juste à temps », les postes ne disposent d'aucune des trois clés du succès : unicité (un seul interlocuteur pour tous les services et destinations, le « one-stop shopping »), fiabilité (une

\footnotetext{
${ }^{49}$ Résolution des directeurs généraux sur l'EMS, Paris, le 20 mars 1987. AN 19990382/14.

50 «Réunion des directeurs généraux. Londres, 22 mai 1987. Création d'une «structure permanente EMS » à Bruxelles. Projet d'ordre du jour ». AN 19990382/14.

${ }^{51}$ EMS International Post Corporation. Société coopérative. Statuts. Projet du 27 août 1987. AN 19990382/14.

${ }^{52}$ D. G. Foot, Royal Mail Letters, 01.09.1987. AN 19990382/14.

${ }^{53}$ Idem.

${ }^{54}$ Jonathan Coopersmith, Faxed. The Rise and Fall of the Fax Machine, Baltimore, The Johns Hopkins University Press, 2015.
} 
Léonard Laborie, « La concurrence avant la libéralisation : International Post Corporation, entreprise de transformation, 1983-1989

seule chaîne de commande, globale, assortie d'un tracing et tracking efficaces), flexibilité (adaptation aux besoins du client). Pour le cabinet, ils est clair enfin que ces marques connues et reconnues ne cessent de prendre du muscle et attaqueront bientôt les marchés domestiques, avec l'aide des autorités, notamment européennes, qui ont déjà marqué leur préférence pour la concurrence. En jeu, ni plus ni moins que « la survie des administrations postales en Europe $»^{55}$.

Les conclusions du rapport sont un véritable électrochoc. La poste britannique les reprend pleinement à son compte. À commencer par la nécessité de créer une structure commerciale internationale unitaire, qui engloberait ce qui est déjà fait pour l'EMS, et irait au-delà pour couvrir tous les segments du marché. Ce changement audacieux est pour elle « essentiel », « vital ». À défaut d'un accord incluant tous les membres de la CEPT, un noyau devrait pouvoir avancer seul, et contracter avec des partenaires privés pour offrir la plus large gamme de services là où les partenaires postaux ne seraient pas mobilisés ${ }^{56}$.

L'administration postale française partage ces vues. Et ose dire ce qui jusqu'alors ne se disait pas : le service international passe après le service national, tout en le finançant ${ }^{57}$. Les modalités de rémunération fixées au sein de l'UPU n'incitent pas au traitement rapide du trafic confié par les postes étrangères ${ }^{58}$, et, par contrecoup, les postes ont tendance à l'exportation à charger lourdement les expéditeurs... Pour ce qui est de l'express, Guy Meynié explique sans détour que via la SFMI, « la France, parce qu'elle souhaite se battre et gagner $»^{59}$, n'hésitera pas à contracter avec des opérateurs privés, en cas de dysfonctionnement, comme c'est souvent le cas en Afrique ou en Amérique latine, ou comme cela arrive lors de mouvements sociaux en Europe. Manière d'encourager les partenaires à se réformer et à aller plus loin dans la coopération?

Pour la Postverket suédoise, seules des entités libérées des entraves politiques pourront mener à bien ces transformations. La priorité est de « donner aux dirigeants de ces entreprises une liberté d'action totale dans des questions de marché et d'exploitation $»^{60}$. Sa proposition d'une déclaration commune de la CEPT en ce sens vise bien sûr les tutelles politiques et les syndicats, qui doivent accepter le changement, mais aussi les responsables des administrations postales eux-mêmes -qui risquent sinon « gérer des organismes à caractère de service public, de mauvais rendement et, par conséquent, soumis à une forte intervention politique ». Face à la gravité de la situation, désormais chiffrée et intégrée, les directeurs décident de mettre sur pied non pas un énième groupe « de travail », mais «d'action », et d'en confier la direction à Sir Ronald Dearing. Dirigeant Royal Mail depuis plusieurs années, mêlant détermination et diplomatie, l'homme est apprécié pour sa droiture et sa modestie -il était connu pour avoir toujours dans sa voiture de la peinture rouge et un pinceau, pour retoucher au passage une éventuelle boîte aux lettres décatie ${ }^{61}$. Proche de Margaret Thatcher, il est surtout reconnu pour avoir réorganisé en profondeur la poste britannique en y insufflant une

\footnotetext{
${ }^{55}$ Résumé et rapport annexé: D. G. Foot, Royal Mail Letters, 01.09.1987. AN 19990382/14. 56 Idem.

${ }^{57}$ Groupe d'Action CEPT, rapport final mai 1988, 93 p., p. 12. AN 19990382/14.

${ }^{58}$ Voir le compte rendu de l'audition de Guy Meynié par Hubert Prévot, le 14 juin 1989, cité ici : https://www.laposte.fr/chp/mediasPdf/NoticesBiographiques/Meynie.pdf (consulté le 28 avril 2021).

${ }^{59}$ Guy Meynié, Réunion des directeurs généraux à Copenhague les 9, 10 et 11 septembre 1987. AN 19990382/14.

${ }^{60}$ Proposition soumise par la Poste de Suède, CEPT, session de Copenhague, 8-17.09.1987. AN 19990382/14

${ }^{61}$ Gerald Kaufman, "Dearing, Ronald Ernest [Ron], Baron Dearinglocked (1930-2009)", Oxford Dictionnary of National Biography, 2013.
} 
Léonard Laborie, « La concurrence avant la libéralisation : International Post Corporation, entreprise de transformation, 1983-1989

culture d'entreprise -sans aller jusqu'à la privatisation, que Thatcher déclare en juin 1987 ne pas envisager ${ }^{62}$. Fort de son expérience, et de sa liberté -il vient d'annoncer qu'il ne ferait pas un nouveau mandat-, Dearing est l'homme de la situation.

Le Groupe d'action commande aussitôt de nouvelles études. Quand l'une explique que le fax et le courrier électronique risquent faire du tort à l'avenir aux coursiers ainsi qu' à l'EMS ${ }^{63}$, l'autre alerte sur le fait que non seulement les postes perdent des parts de marché, mais que ces pertes s'accélèrent ${ }^{64}$. Pour le Groupe d'action, le tableau est « Sinistre. La marche des événements nous balaie ${ }^{65}$.

\section{Le ver est dans le fruit : le repostage 4100}

La situation est d'autant plus inquiétante que les postes sont désunies. Certaines s'allient aux coursiers privés pour capter un surcroit de trafic rémunérateur au détriment de leurs homologues. Les postes néerlandaise et danoise sont régulièrement incriminées pour accepter la remise de courriers originaires par exemple de France, à destination de pays tiers, voire de France même. Cette pratique du repostage ou « remailing » existe de longue date, mais se développe à vive allure depuis la fin des années $1970^{66}$. Les entreprises qui font des envois en masse de relevés ou d'offres commerciales (bulk mail), comme les banques ou les assurances, y trouvent un triple intérêt de réduction des frais de postage, des coûts internes, par la soustraitance du service courrier, et d'accélération de l'acheminement.

En 1987, le groupe de travail dédié de la CEPT conclut que « le remailing pourrait se développer en remplaçant le courrier ordinaire intérieur envoyé par les gros clients ${ }^{67}$. Appelant une réaction « urgente et énergique », il fait trois recommandations. D'abord, changer le système des frais terminaux, c'est-à-dire la manière dont est calculée la rémunération par la poste émettrice de la poste destinatrice du courrier. Ensuite « ne pas démarcher les clients d'une autre administration par des moyens directs ou indirects (filiales à l'étranger) ; ne pas conclure de contrats avec des transporteurs spéculant sur les différences de tarifs appliqués par les administrations ; ne pas fixer des prix internationaux inférieurs aux prix intérieurs, etc. », ce qui en dit long sur la situation. Enfin, créer une sorte d'eurocolis du courrier d'entreprise, baptisé BLS. Les trois recommandations sont approuvées. L'adoption d'une attitude commune est encouragée, mais ce sont les frais terminaux et le service BLS qui pourront concrètement faire l'objet d'accords bilatéraux à compter de janvier 1988.

Dès les premiers accords signés, les « remailers » réagissent. Réunis dans une association qui prend la suite de l'ICC, l'International Express Carriers Conference (IECC), ils déposent en juillet 1988 une plainte auprès de la Commission européenne contre sept postes pour abus de position dominante ${ }^{68}$. Pour l'IECC, « la réexpédition est une joint-venture entre certaines

\footnotetext{
${ }^{62}$ Duncan Campbell-Smith, Masters of the Post. The Authorized History of the Royal Mail, London, Penguin, 2011, p. 600.

${ }^{63}$ PA Consulting Group, «Electronic Information Transmission. Its effect on international mail in the next 10 years", December 1987. AN 19990382/14.

${ }^{64}$ Idem.

${ }^{65}$ Document sans titre ni date, qui cherche à « développer une perception commune des faits. Aider le Groupe d'Action à formuler la réponse à ces faits ». AN 19990382/14.

${ }^{66}$ Campbell, The Rise of Global Delivery Services, .

${ }^{67}$ Rapport du groupe de travail sur les frais terminaux et le nouveau service de courrier d'entreprise, sd. AN 19990382/14.

${ }^{68}$ Requête de l'International Express Carriers Conference en vue d'une procédure en vertu de l'article 3 de la réglementation du conseil n 17 »,13/07/1988. AN 19990382/14.
} 
Léonard Laborie, « La concurrence avant la libéralisation : International Post Corporation, entreprise de transformation, 1983-1989

sociétés de transport express privées et certains services postaux progressistes ${ }^{69}$. Ces accords sont le symptôme du mal (des monopoles inefficaces) autant que son remède (la concurrence). Sondées, les Directions générales XIII (technologies et réseaux de communication) et IV (concurrence) de la Commission européenne déclarent sans détour souhaiter « une suppression complète des monopoles postaux $\gg^{70}$. Les postes remettent en cause la plainte de l'IECC sur le fond comme sur la forme ${ }^{71}$, et commencent à mûrir une position commune sur la question du monopole, plaidant pour son européanisation -formule assez vague, qui laisse des marges de négociation ${ }^{72}$. Les protagonistes se rejoignent en revanche sur la nécessaire autonomisation des postes par rapport aux gouvernements, qui devraient prendre en charge les subventions croisées qu'ils imposent. C'est dans ce contexte que la Commission démarre ses travaux sur le livre vert, privilégiant ce chantier avant de se prononcer sur la question du repostage, à travers un avis préliminaire défavorable aux postes ${ }^{73}$.

\section{«We need to act as one company »}

Le rapport que livre en mai 1988 Ronald Dearing marque l'aboutissement d'un processus démarré en 1984, consistant à forger une vision commune de l'évolution du secteur postal en Europe. Ce n'est plus un cabinet extérieur qui le dit : « les activités internationales des administrations postales sont en grande difficulté et ont perdu une part de marché désastreuse. (...) les transporteurs physiques utilisent la faiblesse du marché international pour développer une énorme force financière et commerciale. (...) cette montée en puissance se répercute sur les marchés intérieurs $\gg^{74}$. C'est sur le créneau du courrier accéléré international que la situation est la plus préoccupante : «Les postes ont dormi pendant que les courriers se sont appropriés $96 \%$ du marché des envois urgents $\gg^{75}$. Mais tout n'est pas perdu, et ce qu'a fait la France avec la SFMI est riche d'espoir ${ }^{76}$. Quatre points faibles sont identifiés : des frais terminaux insuffisants, des quotes-parts pour les colis postaux au contraire exagérées, des procédures douanières à revoir et une « impossibilité d'agir avec une rapidité qui s'adapte aux besoins des clients $»^{77}$.

C'est sur ce dernier point que se concentre le rapport. Il faut selon lui créer une « agence » unique pour concentrer les moyens de renforcer la « force compétitive » des participants ${ }^{78}$.

Rattachée à la CEPT tout en étant ouverte à des postes extra-européennes, elle aurait à la fois des fonctions de prestations de services pour ses membres (étude marketing, étude d'un système informatique intégré) et des fonctions commerciales. Ses missions seraient très larges : pilotage du hub de Bruxelles, et donc de l'EMS - IPC, qui aurait le statut de filiale, élaboration de normes de qualité de service, contractualisation avec chaque poste nationale

\footnotetext{
${ }^{69}$ Idem.

${ }^{70}$ Propos de M. Schuringa. Compte rendu de la première réunion du groupe de travail de la CEPT sur les relations avec la CEE, Londres, 04/10/1988. AN 19990382/14.

71 « Note pour M. René Limat, directeur des services courrier», 29/09/1988. AN 19990382/14.

${ }^{72}$ C. H. Briscoe, « Relations CEPT/CE : document sur les services postaux et 1992 », 08/09/1988. AN 19990382/14.

${ }^{73}$ Campbell, The Rise of Global Delivery Services, p. 273. Voir aussi Kevin Brown, "The big sort out soon to come", Financial Times, 13/04/1990.

${ }^{74}$ Traduction de l'auteur. CEPT Action Group. Consultation Draft Report. Summary, non dated. AN 19990382/14.

75 Idem.

${ }^{76}$ Idem.

${ }^{77}$ Idem.

${ }^{78}$ Groupe d'Action CEPT, rapport final mai 1988, 93 p. AN 19990382/14.
} 
Léonard Laborie, « La concurrence avant la libéralisation : International Post Corporation, entreprise de transformation, 1983-1989

pour leur mise en œuvre et pour la tarification, mesure et contrôle du respect de ces normes jusque chez les exploitants, signature de contrats avec les grands comptes. Les poste resteraient souveraines chacune chez elles, mais devraient consentir à abandonner le contrôle de leurs services internationaux. L'agence devrait d'ailleurs pouvoir mettre à l'amende les membres incapables de se conformer à leurs engagements, voire leur substituer une entreprise privée. Elle aurait donc un pouvoir de contrainte radicalement nouveau. Elle devrait aussi trouver des solutions « concurrentielles » au problème du repostage, avant que cela ne dégénère en « guerre du repostage », interne.

Réunis à Ottawa en mai 1988, les directeurs se divisent. Ceux de Grèce, d'Italie, de Suisse ou de Belgique s'inquiètent de l'ampleur que le projet a prise ${ }^{79}$. Pour la poste irlandaise au contraire « déçue après avoir entendu les doutes exprimés par certains pays. (...) Le temps est venu de vaincre la crainte du changement et de créer l'Agence ${ }^{80}$. Au terme d'une réunion « historique » selon Ronald Dearing, vingt postes décident finalement de prendre part à la fondation d'une agence commune, dotée d'un budget significatif. Parmi les douze de la Communauté économique européenne, seule manque la poste néerlandaise, alors en plein changement de statut. Elles sont rejointes par les postes du Canada, de l'Australie, des EtatsUnis et du Japon. Sachant qu'EMS - IPC doit être avalée d'ici un an, il est décidé de garder ce nom pour celle qu' on dénomme «Agence CEPT International Post Corporation ».

Une fois le principe de la création arrêté, les questions qui se posent sont très concrètes : quel statut pour la nouvelle entité, quel siège, quelle direction ? Les deux premiers sont liés car le champ des possibles change d'un pays à l'autre. La non-participation de la poste néerlandaise exclut Amsterdam. Trois tours de vote sont nécessaires pour imposer Bruxelles, qui l'emporte sur Londres. Le statut qui en découle est soit la coopérative, soit la société anonyme. Quant à la direction, elle sera assurée par un conseil exécutif, chargé de nommer un directeur général. Plusieurs candidats venus des postes nationales et de l'extérieur se présentent. En reconnaissance du rôle moteur joué par l'administration française, qui s'est dotée d'une filiale prise pour modèle, le choix s'arrête sur Guy Meynié ${ }^{81}$. Sa boussole : satisfaire le client, " pratiquer des prix compétitifs, plus étroitement liés aux coûts » ${ }^{82}$, comme on l'explique à la Commission européenne, en somme, transformer les postes nationales en entreprises par leurs relations internationales, débarrassées des tutelles politiques qui créent des distorsions du marché ${ }^{83}$. L'Agence IPC est d'ailleurs hors de portée des gouvernements, qui n'ont peut-être pas toujours été consultés, et qui n'y sont pas représentés ${ }^{84}$.

Le rapport Dearing avait préconisé de démarrer l'agence le plus vite possible, dès septembre 1988. Les discussions qui se prolongent après Ottawa conduisent à un report au début de l'année 1989. " Pour faire en sorte que les Postes membres s'impliquent au plus haut degré », explique une note française ${ }^{85}$, et surtout pour résoudre l'épineux problème juridique qu'il posait, le principe de créer une société dans le cadre d'une association internationale (la CEPT), est abandonné. Une société holding voit le jour, CV International Post Corporation

\footnotetext{
${ }^{79}$ CEPT, Réunion des directeurs généraux des Postes, Ottawa, 19-20 mai 1988, « Compte rendu succinct ». AN 19990382/14.

${ }^{80}$ Idem.

${ }^{81}$ Guy Meynié, “L'agence internationale de coordination postale”, Bulletin de l'IREPP, mars 1989, p. 114-15.

${ }^{82}$ C. H. Briscoe, «Relations CEPT/CE : document sur les services postaux et 1992 », 08/09/1988. AN 19990382/14.

${ }^{83}$ Idem.

${ }^{84}$ Campbell, The Rise of the Global Delivery Services, p. 492.

85 « Evolution de la structure légale et rôle du comité des utilisateurs », sans date ni auteur. AN 19990382/14.
} 
Léonard Laborie, « La concurrence avant la libéralisation : International Post Corporation, entreprise de transformation, 1983-1989

UA, finalement enregistrée à Amsterdam, malgré l'absence de la poste néerlandaise -avec laquelle le torchon brûle sur la question du repostage ${ }^{86}$-, chapeautant non pas une mais deux filiales opérant à Bruxelles. La première est l'EMS- IPC, qui devient une société anonyme ${ }^{87}$. La seconde est une nouvelle société, elle aussi dénommée International Post Corporation... qui reprend les missions de l'agence, en particulier pour l'EMS ${ }^{88}$. Pour éviter la confusion, elle est rebaptisée Unipost à la fin de l'année 1989. Mettant l'accent sur l'unité, ce nom correspond à l'état d'esprit que souhaite insuffler le président du conseil d'administration de la holding, le directeur de l'AN Post irlandaise Gerard Harvey : «we need to act as one company ${ }^{89}$. Pour Harvey, les maîtres mots de l'entreprise sont intégration et coercion : " Market share improvement can only be the result of a wholly professional approach taken by an integrated and effectively managed machine applying all the appropriate disciplines and sanctions which are exercised by any successful international business organization" ${ }^{90}$. Les "sanctions" envisagées et notamment la capacité de ne pas confier le courrier qui lui reviendrait à l'un des membres même du consortium attirent aussitôt les remontrances du gardien du temple postal, le bureau international de l'UPU qui estime cette mesure fort « dangereuse $»^{91}$.

Prometteur mais complexe sur la forme, l'ensemble du dispositif IPC est surtout problématique sur le fond : les actionnaires se mettent, individuellement, dans la position d'être exclu du marché par leur propre filiale collective. Cela n'empêche pas un certain nombre de postes, la Royal Mail britannique la première, d'effectivement signer un contrat avec IPC dès les premiers mois de 1989,. « I am quite content for IPC to dictate the system that we should adopt", déclare Nick Nelson, patron de Royal Mail Parcels ${ }^{22}$.

4. Conclusion

Les responsables des postes n'ont pas attendu le livre vert sur le développement du marché unique des services postaux, publié en 1992 par la Commission européenne, ni même, dans le but d'influencer cette dernière, la création sous la houlette des ministres d'un Senior Official Group - Post (SOGP) fin 1989, pour penser la concurrence et la vivre au point même de sentir leurs organisations mourir. La pression concurrentielle est pour eux sensible bien avant la libéralisation communautaire.

C'est ensemble qu'ils forgent cette vision apocalyptique du monde postal durant les années 1980. Et c'est ensemble qu'ils cherchent des solutions. Le but est double : d'une part préserver l'ordre international séculaire de postes souveraines et non concurrentes, d'autre part transformer chacune en entreprise plus agile capable de répondre à la concurrence des

86 « Poste : la main dans le sac. Du courrier français transite par les Pays-Bas », Le Point, 08/05/1989.

${ }^{87}$ Guy Meynié, directeur de l'Agence CEPT IPC, à M. Delage, directeur général de la Poste, Bruxelles, 06/03/1989. AN 19990382/14.

${ }^{88}$ Paul Abrahams, « Parcelforce leads the counter-attack”, Financial Times, 22/06/1990.

${ }^{89}$ Postal Life, vol. 26, n5, 1991, p. 14.

${ }^{90}$ Gerard Harvey, « Unipost. A Postal Strategy in a Global Market”, in Walpurga Speckbacher (éd.), Die Zukunft der Postdienste in Europa: Proceedings der internationalen Konferenz „Die Zukunft der Postdienste in Europa“ Bonn, 25.-26.10.1990, Springer, 2013, p. 179-184, p. 184.

${ }^{91}$ Conseil exécutif de l'UPU, « La menace de la concurrence : la CEPT s'adapte à l'évolution du marché. (Point 20 de l'ordre du jour). Exposé oral du secrétaire général : Commentaires à propos du rapport présenté par l'Administration gérante de la CEPT », sd. AN 19990382/14. 92 Kevin Willmott, "Postal co-operation eyes international parcels market", Freight Management, vol. 23, n²67, June 1989, p. 8. 
Léonard Laborie, « La concurrence avant la libéralisation : International Post Corporation, entreprise de transformation, 1983-1989

opérateurs privés sur des marchés déjà ouverts et qui s'inventent (le colis, l'express, le courrier électronique). La poste française joue dans ce processus un rôle moteur. La création de la holding International Post Corporation et de la société Unipost marquent le passage d'une logique de cartel administratif, à une logique d'entreprise, tournée vers l'augmentation de ses parts de marché et dotée d'un pouvoir d'initiative et de décision. Au passage, un tabou postal séculaire est levé. Unipost peut contracter avec une entreprise privée pour assurer le service qu'une poste échouerait à rendre efficacement sur son territoire. Si IPC est taillé pour maintenir l'ordre, il menace en même temps de le renverser.

L'entreprise n'est pas sans fragilité. Du fait de la diversité des statuts et des capacités financières, les actionnaires de la holding n'ont au fond ni les mêmes moyens, ni les mêmes ambitions. Comme le montre la pratique du repostage, ils n'ont pas non plus toujours les mêmes intérêts. Dans ces conditions, le processus d'intégration engagé pouvait-il être autre chose qu'une transition?

\section{Epilogue}

Les ambitions placées dans l'IPC sont vite déçues. Quand l'occasion de pousser la dynamique d'entreprise se présente, les partenaires se divisent. Ceci intervient dès 1991. À cette époque, les marges de tous les opérateurs s'effondrent par le jeu de la concurrence, notamment sur le terrain européen où les investissements nécessaires sont énormes ${ }^{93}$. Les intégrateurs qui paraissaient jadis d'une insolente vigueur, des « rouleaux compresseurs » à la « force de frappe tant financière que technique » sans commune mesure connaissent des difficultés ${ }^{94}$. L'essor du fax leur fait mal, le commerce électronique n'a pas encore décollé, les transporteurs routiers s'organisent... et le front uni des postes commence sans doute à faire effet. Il leur faut reprendre leur souffle. DHL se fait rattraper par deux compagnies aériennes, Lufthansa et Japan Airlines, qui prennent une participation majoritaire. Federal Express se demande si elle va rester en Europe et renonce à sponsoriser les Jeux Olympiques d'Albertville et de Barcelone en $1992^{95}$. TNT se tourne vers les postes, qui ne peuvent refuser l'opportunité que représente l'accès à la marque et au réseau aérien de l'entreprise australienne. Mais seules quelques unes se lancent dans cette nouvelle aventure. En 1991, les postes d'Allemagne, du Canada, de France, des Pays-Bas et de Suède, qui sont depuis le début parmi les plus motrices et les moins empêchées, s'allient au sein d'une nouvelle holding ouverte, GDNET, pour créer une joint-venture avec TNT, GDEW ${ }^{96}$. L'échec de GDEW cinq ans plus tard ouvre une période de rachats, de fusions et d'accords de partenariat entre opérateurs publics et privés, formant des groupes en compétition sur le marché européen et mondial ${ }^{97}$. Si International Post Corporation survit à ces évolutions inattendues, elle se concentre sur de discrètes missions d'études ${ }^{98}$. Unipost quant à elle disparaît du paysage. C'est ainsi que toute une expérience courte mais fondatrice de transformation coopérative tombe dans l'oubli, à travers laquelle les postes historiques, qui se voyaient disparaître, se

\footnotetext{
${ }_{93}$ Philippe Moreau, « Messagerie express : la riposte s'organise face aux « integrators » », Les Echos, 31/10/1991.

${ }^{94}$ Martine Robert, «L'offensive des géants du fret express freinée par la riposte des nationaux », Les Echos, 27/02/1992.

${ }^{95}$ Jean-Pierre Bodet, directeur du réseau, « Note pour M. le directeur général de la Poste », 09/02/1989. AN 19990382/14.

${ }^{96}$ Direction de la stratégie et de la planification, « Note à l'attention de Monsieur Veillon. Bilan d'exécution 1991 du contrat de plan », 01/07/1992. AN 20020509/11. Sur l'absence de Royal Mail : Campbell-Smith, Masters of the Post, p. 636-637.

${ }^{97}$ Allaz, Une histoire de la Poste dans le monde, p. 623-627.

98 https://www.ipc.be/about-ipc (consulté le 15 mai 2021).
} 
Léonard Laborie, « La concurrence avant la libéralisation : International Post Corporation, entreprise de transformation, 1983-1989

sont au contraire mutuellement préparées, dans un contexte de grande incertitude réglementaire, commerciale et technique, à devenir des entreprises presque comme les autres, capables de rester actrices de leur destin. 